\title{
MAXIMAL OPERATORS RELATED TO BLOCK SPACES
}

\author{
H. M. Al-QASSEM
}

\begin{abstract}
In this paper, we prove appropriate $L^{p}$ bounds for a class of maximal operators $\mathscr{S}_{\Omega}$ related to singular integrals with kernels which belong to block spaces and are supported by subvarieties. Also, we show that our condition on the kernel is optimal for the $L^{2}$ boundedness of $\mathscr{S}_{\Omega}$. Our results improve substantially the main result obtained by L. K. Chen and H. Lin in $[\mathrm{CL}]$.
\end{abstract}

\section{Introduction and statement of results}

Let $\mathbf{S}^{n-1}$ denote the unit sphere in $\mathbf{R}^{n}(n \geq 2)$ equipped with the normalized Lebesgue measure $d \sigma=d \sigma(\cdot)$. Throughout this paper, $p^{\prime}$ will denote the dual exponent to $p$, that is $1 / p+1 / p^{\prime}=1$. Also, we shall let $\Omega$ be a homogeneous function of degree zero which satisfies $\Omega \in L^{1}\left(\mathbf{S}^{n-1}\right)$ and

$$
\int_{\mathbf{S}^{n-1}} \Omega(u) d \sigma(u)=0 .
$$

Let $\mathscr{H}=$ the set of all radial functions $h$ satisfying

$$
\left(\int_{0}^{\infty}|h(r)|^{2} \frac{d r}{r}\right)^{1 / 2} \leq 1 .
$$

Also, for $d \neq 0$, we say that a smooth function $\Psi: \mathbf{R}_{+} \rightarrow \mathbf{R}$ belongs to the class $\Gamma_{d}\left(\mathbf{R}_{+}\right)$if for some positive constants $C_{1}, C_{2}, C_{3}$, and $C_{4}$ independent of $t$, the following growth conditions are satisfied:

$$
\begin{aligned}
|\Psi(t)| \leq C_{1} t^{d}, \quad\left|\Psi^{\prime \prime}(t)\right| \leq C_{2} t^{d-2}, \\
C_{3} t^{d-1} \leq\left|\Psi^{\prime}(t)\right| \leq C_{4} t^{d-1} .
\end{aligned}
$$

Now, a smooth function $\Psi: \mathbf{R}_{+} \rightarrow \mathbf{R}$ belongs to the class $\Gamma\left(\mathbf{R}_{+}\right)$if $\Psi \in \Gamma_{d}\left(\mathbf{R}_{+}\right)$ for some $d \neq 0$.

For a function $\Psi \in \Gamma\left(\mathbf{R}_{+}\right)$we define the maximal operator $\mathscr{S}_{\Omega, \Psi}$ by

2000 Mathematics Subject Classification. Primary 42B20; Secondary 42B15, 42B25.

Key words and phrases. Maximal operator, Rough kernel, Singular integral, $L^{p}$ boundedness, Block spaces.

Received June 1, 2004; revised January 31, 2005. 


$$
\mathscr{S}_{\Omega, \Psi} f(x)=\left.\sup _{h \in \mathscr{H}}\left|\int_{\mathbf{R}^{n}} f\left(x-\Psi(|y|) y^{\prime}\right) h(|y|) \Omega\left(y^{\prime}\right)\right| y\right|^{-n} d y \mid,
$$

where $y^{\prime}=y /|y| \in \mathbf{S}^{n-1}$ and $f \in \mathscr{S}\left(\mathbf{R}^{n}\right)$, the space of Schwartz functions.

The maximal operator $\mathscr{S}_{\Omega, \Psi}$ is closely related to the singular integral operator $T_{\Psi, \Omega, h}$ given by

$$
T_{\Psi, \Omega, h} f(x)=p . v \cdot \int_{\mathbf{R}^{n}} f\left(x-\Psi(|y|) y^{\prime}\right) \frac{\Omega\left(y^{\prime}\right)}{|y|^{n}} h(|y|) d y,
$$

where $h$ is a measurable function on $\mathbf{R}_{+}$.

For the sake of simplicity, we denote $\mathscr{S}_{\Omega, \Psi}=\mathscr{S}_{\Omega}$ and $T_{\Psi, \Omega, h}=T_{\Omega, h}$ if $\Psi(t)=t$.

In $[\mathrm{CL}], \mathrm{L} . \mathrm{K}$. Chen and H. Lin studied the $L^{p}$ boundedness of the maximal operator $\mathscr{S}_{\Omega}$ under a smoothness condition on $\Omega$. In fact, they proved the following:

Theorem A [CL]. Assume $n \geq 2$ and $\Omega \in C\left(\mathbf{S}^{n-1}\right)$ satisfying (1.1). Then

$$
\left\|\mathscr{S}_{\Omega}(f)\right\|_{L^{p}\left(\mathbf{R}^{n}\right)} \leq C_{p}\|f\|_{L^{p}\left(\mathbf{R}^{n}\right)}
$$

for $2 n /(2 n-1)<p<\infty$ and $f \in L^{p}$. Moreover, the range of $p$ is the best possible.

On the other hand, the $L^{p}$ boundedness of the singular integral operator $T_{\Psi, \Omega, h}$ is known to hold under much weaker conditions on $\Omega$ (see $[\mathrm{CZ}$, $[\mathrm{Fe}],[\mathrm{DR}],[\mathrm{LTW}],[\mathrm{FP}],[\mathrm{AA} 1],[\mathrm{AP}])$. For example, if $\Omega \in L \log ^{+} L\left(\mathbf{S}^{n-1}\right)$, Calderón-Zygmund showed that $T_{\Omega, 1}$ is bounded on $L^{p}$ for all $p \in(1, \infty)$ and the condition $\Omega \in L \log ^{+} L\left(\mathbf{S}^{n-1}\right)$ is essentially the weakest possible size condition on $\Omega$ for the $L^{p}$ boundedness of $T_{\Omega, 1}$ to hold ([CZ]). Some years later, Connett ([Co]) and Coifman-Weiss $([\mathrm{CW}])$ obtained an improvement over the result of Calderón and Zygmund by considering $\Omega$ in the Hardy space $H^{1}\left(\mathbf{S}^{n-1}\right)$. The study of the $L^{p} \quad(1<p<\infty)$ boundedness of the singular operator $T_{\Omega, h}$ began in R. Fefferman in [Fe] if $h \in L^{\infty}\left(\mathbf{R}^{+}\right)$and $\Omega$ satisfies some Lipschitz condition on $S^{n-1}$ and subsequently by many authors under various conditions on $\Omega$ and $h$ (see for example, [Na], [Ch], [DR]). In 1997, Fan and Pan introduced the more general class of operators $T_{\Psi, \Omega, h}$ and showed that $T_{\Psi, \Omega, h}$ is bounded on $L^{p}\left(\mathbf{R}^{n}\right)$ $(1<p<\infty)$ if $H^{1}\left(\mathbf{S}^{n-1}\right)$ and $h \in L^{\infty}\left(\mathbf{R}_{+}\right)$. Another condition on $\Omega$ was given by Jiang and Lu who introduced a special class of block spaces $B_{q}^{(0, v)}\left(\mathbf{S}^{n-1}\right)$ and proved the following $L^{2}$ boundedness result.

THEOREM B ([LTW]). Let $T_{\Omega, h}$ be given as above. Then if $h \in L^{\infty}\left(\mathbf{R}_{+}\right)$and $\Omega \in B_{q}^{(0,0)}\left(\mathbf{S}^{n-1}\right)$ with $q>1, T_{\Omega, h}$ is a bounded operator on $L^{2}\left(\mathbf{R}^{n}\right)$.

Some years later, the $L^{p}$ boundedness of the more general operator $T_{\Psi, \Omega, h}$ was proved for all $p \in(1, \infty)$ under the condition $\Omega \in B_{q}^{(0,0)}\left(\mathbf{S}^{n-1}\right)$ (see for example, [AA1]). Also, it was proved in [AAP1] that the condition $\Omega \in B_{q}^{(0,0)}\left(\mathbf{S}^{n-1}\right)$ 
is the best possible for the $L^{p}$ boundedness of $T_{\Omega, 1}$ to hold. Namely, the $L^{p}$ boundedness of $T_{\Omega, 1}$ may fail for any $p$ if it is replaced by a weaker condition $\Omega \in B_{q}^{(0, v)}\left(\mathbf{S}^{n-1}\right)$ for any $-1<v<0$ and $q>1$. The definition of the block space $B_{q}^{(0, v)}\left(\mathbf{S}^{n-1}\right)$ will be recalled in Section 2 .

The results cited in [AA1] and [AAP1] above on singular integrals give rise to the problem whether similar results hold for the maximal integral operator $\mathscr{S}_{\Omega, \Psi}$. More precisely, we have the following:

Problem. Determine wether the $L^{p}$ boundedness of the operator $\mathscr{S}_{\Omega}$ holds under a condition in the form of $\Omega \in B_{q}^{(0, v)}\left(\mathbf{S}^{n-1}\right),-1<v$, and, if so, what is the best possible value of $v$.

The main focus of this paper is to obtain a solution to the above problem. Our main result in this paper is the following:

THEOREM 1.1. Let $n \geq 2$ and $\mathscr{S}_{\Omega, \Psi}$ be given as in (1.4). Then

(a) If $\Omega \in B_{q}^{(0,-1 / 2)}\left(\mathbf{S}^{n-1}\right), q>1$, and satisfies (1.1), $\mathscr{S}_{\Omega, \Psi}$ is bounded on $L^{p}\left(\mathbf{R}^{n}\right)$ for $2 \leq p<\infty$;

(b) If $\Omega \in L^{q}\left(\mathbf{S}^{n-1}\right)$ (for some $q>1$ ) and satisfies (1.1), $\mathscr{S}_{\Omega, \Psi}$ is bounded on $L^{p}\left(\mathbf{R}^{n}\right)$ for $2 n \delta /(2 n+n \delta-2)<p<\infty$, where $\delta=\max \left\{2, q^{\prime}\right\}$.

(c) There exists an $\Omega$ which lies in $B_{q}^{(0, v)}\left(\mathbf{S}^{n-1}\right)$ for all $-1<v<-\frac{1}{2}$ and satisfies (1.1) such that $\mathscr{S}_{\Omega}$ is not bounded on $L^{2}\left(\mathbf{R}^{n}\right)$.

We remark that on $\mathbf{S}^{n-1}$, for any $q>1$ and $-1<v$, the following inclusions hold and are proper:

$$
C^{1}\left(\mathbf{S}^{n-1}\right) \subset \bigcup_{r>1} L^{r}\left(\mathbf{S}^{n-1}\right) \subset B_{q}^{(0, v)}\left(\mathbf{S}^{n-1}\right) .
$$

By the relationship in (1.6) remarked above one sees that parts (a) and (b) represent a substantial improvement of the main result of L. K. Chen and H. Lin, while part (c) shows that the condition $\Omega \in B_{q}^{(0,-1 / 2)}\left(\mathbf{S}^{n-1}\right)$ is nearly optimal.

Throughout the rest of the paper the letter $C$ will stand for a constant but not necessarily the same one in each occurrence.

Acknowledgment. The author would like to thank very much the referee for his very valuable comments and suggestions.

\section{Definitions and lemmas}

The block spaces originated in the work of M. H. Taibleson and G. Weiss on the convergence of the Fourier series (see [TW]) in connection with the developments of the real Hardy spaces. Below we shall recall the definition of block spaces on $\mathbf{S}^{n-1}$. For further background information about the theory of spaces generated by blocks and its applications to harmonic analysis, see the book [LTW]. 
Definition 2.1. A q-block on $\mathbf{S}^{n-1}$ is an $L^{q}(1<q \leq \infty)$ function $b(x)$ that satisfies
(i) $\operatorname{supp}(b) \subset I$;
(ii) $\|b\|_{L^{q}} \leq|I|^{-1 / q^{\prime}}$,

where $|I|=\sigma(I)$, and $I=B\left(x_{0}^{\prime}, \theta_{0}\right)=\left\{x^{\prime} \in \mathbf{S}^{n-1}:\left|x^{\prime}-x_{0}^{\prime}\right|<\theta_{0}\right\}$ is a cap on $\mathbf{S}^{n-1}$ for some $x_{0}^{\prime} \in \mathbf{S}^{n-1}$ and $\theta_{0} \in(0,1]$.

Jiang and $\mathrm{Lu}$ introduced (see [LTW]) the class of block spaces $B_{q}^{(0, v)}\left(\mathbf{S}^{n-1}\right)$ (for $v>-1$ ) with respect to the study of the singular integral operators $T_{\Omega, h}$.

Definition 2.2. The block space $B_{q}^{(0, v)}\left(\mathbf{S}^{n-1}\right)$ is defined by

$$
B_{q}^{(0, v)}\left(\mathbf{S}^{n-1}\right)=\left\{\Omega \in L^{1}\left(\mathbf{S}^{n-1}\right): \Omega=\sum_{\mu=1}^{\infty} \lambda_{\mu} b_{\mu}, M_{q}^{(0, v)}\left(\left\{\lambda_{\mu}\right\}\right)<\infty\right\},
$$

where each $\lambda_{\mu}$ is a complex number; each $b_{\mu}$ is a q-block supported on a cap $I_{\mu}$ on $\mathbf{S}^{n-1}, v>-1$ and

$$
M_{q}^{(0, v)}\left(\left\{\lambda_{\mu}\right\}\right)=\sum_{\mu=1}^{\infty}\left|\lambda_{\mu}\right|\left\{1+\log ^{(v+1)}\left(\left|I_{\mu}\right|^{-1}\right)\right\} .
$$

We remark that the definition of $B_{q}^{(0, v)}([a, b]), a, b \in \mathbf{R}$ will be the same as that of $B_{q}^{(0, v)}\left(\mathbf{S}^{n-1}\right)$ except for minor modifications.

Let $\|\Omega\|_{B_{q}^{(0, v)}\left(\mathbf{S}^{n-1}\right)}=N_{q}^{(0, v)}(\Omega)=\inf \left\{M_{q}^{(0, v)}\left(\left\{\lambda_{\mu}\right\}\right): \Omega=\sum_{\mu=1}^{\infty} \lambda_{\mu} b_{\mu}\right.$ and each $b_{\mu}$ is a $q$-block function supported on a cap $I_{\mu}$ on $\left.\mathbf{S}^{n-1}\right\}$. Then $\|\cdot\|_{B_{q}^{(0, v)}\left(\mathbf{S}^{n-1}\right)}$ is a norm on the space $B_{q}^{(0, v)}\left(\mathbf{S}^{n-1}\right)$ and $\left(B_{q}^{(0, v)}\left(\mathbf{S}^{n-1}\right),\|\cdot\|_{B_{q}^{(0, v)}\left(\mathbf{S}^{n-1}\right)}\right)$ is a Banach space.

In their investigations of block spaces, Keitoku and Sato in [KS] showed that these spaces enjoy the following properties:

$$
\begin{aligned}
B_{q}^{\left(0, v_{2}\right)}\left(\mathbf{S}^{n-1}\right) & \subset B_{q}^{\left(0, v_{1}\right)}\left(\mathbf{S}^{n-1}\right) \quad \text { if } v_{2}>v_{1}>-1 ; \\
B_{q_{2}}^{(0, v)}\left(\mathbf{S}^{n-1}\right) & \subset B_{q_{1}}^{(0, v)}\left(\mathbf{S}^{n-1}\right) \quad \text { if } 1<q_{1}<q_{2} \text { and for any } v>-1 ; \\
\bigcup_{q>1} B_{q}^{(0, v)}\left(\mathbf{S}^{n-1}\right) & \varsubsetneqq \bigcup_{q>1} L^{q}\left(\mathbf{S}^{n-1}\right) \quad \text { for any } v>-1 .
\end{aligned}
$$

The proof of Theorem 1.1 (c) will rely heavily on the following lemma from [AAP1].

Lemma 2.3. For any $v>-1, a, b \in \mathbf{R}$,

(i) If $f \in B_{q}^{(0, v)}([a, b])$ and $g$ is a measurable on $[a, b]$ with $|g| \leq|f|$, then $g \in B_{q}^{(0, v)}([a, b])$ with

$$
N_{q}^{(0, v)}(g) \leq N_{q}^{(0, v)}(f)
$$


(ii) Let $I_{1}$ and $I_{2}$ be two disjoint intervals in $[a, b]$ with $\left|I_{1}\right|,\left|I_{2}\right|<1$ and $\alpha_{1}, \alpha_{2} \in \mathbf{R}^{+}$. Then

$$
N_{q}^{(0, v)}\left(\alpha_{1} \chi_{I_{1}}+\alpha_{2} \chi_{I_{2}}\right) \geq N_{q}^{(0, v)}\left(\alpha_{1} \chi_{I_{1}}\right)+N_{q}^{(0, v)}\left(\alpha_{2} \chi_{I_{2}}\right) ;
$$

(iii) Let $I$ be an interval in $[a, b]$ with $|I|<1$. Then

$$
N_{q}^{(0, v)}\left(\chi_{I}\right) \geq|I|\left(1+\log ^{v+1}\left(|I|^{-1}\right)\right) .
$$

Lemma 2.4. Let $q>1, \mu \in \mathbf{N} \cup\{0\}$ and $\tilde{b}_{\mu}$ be a function on $\mathbf{S}^{n-1}$ satisfying (i) $\int_{\mathbf{S}^{n-1}} \tilde{b}_{\mu}(y) d \sigma(y)=0$; (ii) $\left\|\tilde{b}_{\mu}\right\|_{q} \leq\left|I_{\mu}\right|^{-1 / q^{\prime}}$ for some cap $I_{\mu}$ on $\mathbf{S}^{n-1}$ with $\left|I_{\mu}\right|<e^{-2}$; and (iii) $\left\|\tilde{b}_{\mu}\right\|_{1} \leq 1$. Assume that $\Psi$ belongs to the class $\Gamma\left(\mathbf{R}_{+}\right)$for some $d \neq 0$. Let $\omega_{\mu}=2^{\log \left(\left|I_{\mu}\right|^{-1}\right)}$ and for $\xi \in \mathbf{R}^{n}$, let

$$
Y_{\mu, k}(\xi)=\left(\int_{\omega_{\mu}^{k}}^{\omega_{\mu}^{k+1}}\left|\int_{\mathbf{S}^{n-1}} \tilde{b}_{\mu}(x) e^{-i \Psi(t)(\xi \cdot x)} d \sigma(x)\right|^{2} \frac{d t}{t}\right)^{1 / 2} .
$$

Then there exist positive constants $C$ and $\alpha$ with $0<\alpha q^{\prime}<1$ such that

$$
\begin{aligned}
& \left|Y_{\mu, k}(\xi)\right| \leq C\left(\log \left|I_{\mu}\right|^{-1}\right)^{1 / 2} \\
& \left|Y_{\mu, k}(\xi)\right| \leq C\left(\log \left|I_{\mu}\right|^{-1}\right)^{1 / 2}\left|\omega_{\mu}^{k d} \xi\right|^{ \pm \alpha / \log \left(\left|I_{\mu}\right|^{-1}\right)},
\end{aligned}
$$

where $t^{ \pm \alpha}=\inf \left\{t^{\alpha}, t^{-\alpha}\right\}$ and $C$ is a positive constant independent of $k, \xi$ and $\mu$.

Proof. We shall prove our estimates only for the case $d>0$, because the proof for the case $d<0$ is essentially the same. First, by condition (iii), it is easy to verify that $(2.8)$ holds.

Next, since

$$
\begin{aligned}
& \left|\int_{\mathbf{S}^{n-1}} \tilde{b}_{\mu}(x) e^{-i \Psi(t)(\xi \cdot x)} d \sigma(x)\right|^{2} \\
& \quad=\int_{\mathbf{S}^{n-1} \times \mathbf{S}^{n-1}} \tilde{b}_{\mu}(x) \overline{\tilde{b}_{\mu}(y)} e^{-i \Psi(t) \xi(x-y)} d \sigma(x) d \sigma(y)
\end{aligned}
$$

we get

$$
\begin{aligned}
& \left|Y_{\mu, k}(\xi)\right|^{2} \\
& \quad=\int_{\mathbf{S}^{n-1} \times \mathbf{S}^{n-1}} \tilde{b}_{\mu}(x) \overline{\tilde{b}_{\mu}(y)}\left(\int_{1}^{\omega_{\mu}} e^{-i \Psi\left(\omega_{\mu}^{k} t\right) \xi \cdot(x-y)} \frac{d t}{t}\right) d \sigma(x) d \sigma(y) .
\end{aligned}
$$

By integration by parts, it is easy to verify that

$$
\left|\int_{1}^{\omega_{\mu}} e^{-i \Psi\left(\omega_{\mu}^{k} t\right) \xi \cdot(x-y)} \frac{d t}{t}\right| \leq C \omega_{\mu}\left|\omega_{\mu}^{k d} \xi\right|^{-1}\left|\xi^{\prime} \cdot(y-x)\right|^{-1}
$$

which when combined with the trivial estimate 


$$
\left|\int_{1}^{\omega_{\mu}} e^{-i \Psi\left(\omega_{\mu}^{k} t\right) \xi \cdot(x-y)} \frac{d t}{t}\right| \leq C\left(\log \left|I_{\mu}\right|^{-1}\right)
$$

yields

$$
\begin{aligned}
& \left|\int_{1}^{\omega_{\mu}} e^{-i \Psi\left(\omega_{\mu}^{k} t\right) \xi \cdot(x-y)} \frac{d t}{t}\right| \\
& \quad \leq C\left(\log \left|I_{\mu}\right|^{-1}\right) \omega_{\mu}^{\alpha}\left|\omega_{\mu}^{k d} \xi\right|^{-\alpha}\left|\xi^{\prime} \cdot(y-x)\right|^{-\alpha}
\end{aligned}
$$

for any $0<\alpha<1$.

By Hölder's inequality, condition (ii) on $\tilde{b}_{\mu}$ and $(2.10)-(2.11)$ we get

$$
\begin{aligned}
& \left|Y_{\mu, k}(\xi)\right| \\
& \quad \leq C\left(\log \left|I_{\mu}\right|^{-1}\right)^{1 / 2} \omega_{\mu}^{\alpha / 2}\left|\omega_{\mu}^{k d} \xi\right|^{-\alpha / 2}\left(\int_{\mathbf{S}^{n-1} \times \mathbf{S}^{n-1}}\left|\xi^{\prime} \cdot(x-y)\right|^{-\alpha q^{\prime}} d \sigma(x) d \sigma(y)\right)^{1 / 2}
\end{aligned}
$$

By choosing $\alpha q^{\prime}<1$, we get

$$
\left|Y_{\mu, k}(\xi)\right| \leq C\left(\log \left|I_{\mu}\right|^{-1}\right)^{1 / 2} \omega_{\mu}^{\alpha / 2}\left|\omega_{\mu}^{k d} \xi\right|^{-\alpha / 2}
$$

which, when combined with the trivial estimate (2.8), yields the estimate (2.9) with a minus sign in the exponent. To get the second estimate, we use the cancellation condition (i) on $\tilde{b}_{\mu}$ to get

$$
\left|Y_{\mu, k}(\xi)\right|^{2} \leq \int_{1}^{\omega_{\mu}}\left(\int_{\mathbf{S}^{n-1}}\left|\tilde{b}_{\mu}(x)\right|\left|e^{-i \Psi\left(\omega_{\mu}^{k} t\right) \xi \cdot x}-1\right| d \sigma(x)\right)^{2} \frac{d t}{t}
$$

By (1.2) and condition (iii), we get

$$
\left|Y_{\mu, k}(\xi)\right| \leq C\left(\log \left|I_{\mu}\right|^{-1}\right)^{1 / 2}\left|\omega_{\mu}^{k d} \xi\right| .
$$

By interpolation between this estimate with the trivial estimate (2.8) we get the second estimate in (2.9). This completes the proof of the lemma.

By the proof of Lemma 3.1 in [AAP2], we get the following:

Lemma 2.5. Let $\left\{v_{k}: k \in \mathbf{Z}\right\}$ be a sequence of non negative Borel measures on $\mathbf{R}^{n}$. Suppose that for all $k \in \mathbf{Z}, \xi \in \mathbf{R}^{n}$, for some $a \geq 2, \alpha, C>0$ and for some constant $B>1$ we have

(i) $\left\|v_{k}\right\| \leq B$

(ii) $\left|\hat{v}_{k}(\xi)\right| \leq C B\left|a^{k B} \xi\right|^{-\alpha / B}$;

(iii) $\left|\hat{v}_{k}(\xi)-1\right| \leq C B\left|a^{k B} \xi\right|^{\alpha / B}$.

Then the inequality

$$
\left\|v^{*}(f)\right\|_{p} \leq C B\|f\|_{p}
$$
holds for all $1<p \leq \infty$ and $f$ in $L^{p}\left(\mathbf{R}^{n}\right)$, where $v^{*}(f)=\sup _{k \in \mathbf{Z}}\left|v_{k} * f\right|$. The
constant $C_{p}$ is independent of $B$. 
For any $\mu \in \mathbf{N}$ and $\tilde{b}_{\mu} \in L^{1}\left(\mathbf{S}^{n-1}\right)$, we define the maximal operator

$$
v_{\mu}^{*} f(x)=\sup _{k \in \mathbf{Z}}\left|\int_{\omega_{\mu}^{k} \leq|y|<\omega_{\mu}^{k+1}} f\left(x-\Psi(|y|) y^{\prime}\right) \frac{\tilde{b}_{\mu}\left(y^{\prime}\right)}{|y|^{n}} d y\right|,
$$

where $\omega_{\mu}=2^{\log \left(\left|I_{\mu}\right|^{-1}\right)}$ for some cap $I_{\mu}$ on $\mathbf{S}^{n-1}$.

By Lemmas 2.4 and 2.5, we get immediately the following:

Lemma 2.6. Let $\mu \in \mathbf{N}$ and let $\tilde{b}_{\mu}$ be a function on $\mathbf{S}^{n-1}$ satisfying (i) $\left\|\tilde{b}_{\mu}\right\|_{q} \leq\left|I_{\mu}\right|^{-1 / q^{\prime}}$ for some $q>1$ and for some cap $I_{\mu}$ on $\mathbf{S}^{n-1}$; (ii) $\left\|\tilde{b}_{\mu}\right\|_{1} \leq 1$. Assume that $\Psi$ belongs to the class $\Gamma\left(\mathbf{R}_{+}\right)$for some $d \neq 0$. Then

$$
\left\|v_{\mu}^{*}(f)\right\|_{p} \leq C_{p}\left(\log \left|I_{\mu}\right|^{-1}\right)\|f\|_{p}
$$

for $1<p \leq \infty$ and $f \in L^{p}$, where $C_{p}$ is independent of $\mu$ and $f$.

Let $\mathscr{M}_{S}$ be the spherical maximal operator defined by

$$
\mathscr{M}_{S} f(x)=\sup _{r>0} \int_{\mathbf{S}^{n-1}}|f(x-r \theta)| d \sigma(\theta) .
$$

By the results of E. M. Stein [St3] and J. Bourgain [Bo] we have

Lemma 2.7. Suppose that $n \geq 2$ and $p>n /(n-1)$. Then $\mathscr{M}_{S}(f)$ is bounded on $L^{p}\left(\mathbf{R}^{n}\right)$.

\section{Proof of the main result}

Proof of Theorem 1.1 (a). Assume that $\Omega \in B_{q}^{(0,-1 / 2)}\left(\mathbf{S}^{n-1}\right)$ for some $q>1$ and satisfies (1.1). Thus $\Omega$ can be written as $\Omega=\sum_{\mu=1}^{\infty} \lambda_{\mu} b_{\mu}$, where $\lambda_{\mu} \in \mathbf{C}, b_{\mu}$ is a $q$-block supported on a cap $I_{\mu}$ on $\mathbf{S}^{n-1}$ and $M_{q}^{(0,-1 / 2)}\left(\left\{\lambda_{\mu}\right\}\right)<\infty$. To each block function $b_{\mu}(\cdot)$, let $\tilde{b}_{\mu}(\cdot)$ be a function defined by

$$
\tilde{b}_{\mu}(x)=b_{\mu}(x)-\int_{\mathbf{S}^{n-1}} b_{\mu}(u) d \sigma(u) .
$$

Let $\mathbf{J}=\left\{\mu \in \mathbf{N}:\left|I_{\mu}\right|<e^{-2}\right\} . \quad$ Let $\tilde{b}_{0}=\Omega-\sum_{\mu \in \mathbf{J}}^{\infty} \lambda_{\mu} \tilde{b}_{\mu}$. Then for some positive constant $C$, the following holds for all $\mu \in \mathbf{J} \cup\{0\}$ :

$$
\begin{aligned}
\int_{\mathbf{S}^{n-1}} \tilde{b}_{\mu}(u) d \sigma(u) & =0, \\
\left\|\tilde{b}_{\mu}\right\|_{q} & \leq C\left|I_{\mu}\right|^{-1 / q^{\prime}}, \\
\left\|\tilde{b}_{\mu}\right\|_{1} & \leq C, \\
\Omega & =\sum_{\mu \in \mathbf{J} \cup\{0\}} \lambda_{\mu} \tilde{b}_{\mu},
\end{aligned}
$$


where $I_{0}$ is any cap on $\mathbf{S}^{n-1}$ with $\left|I_{0}\right|=e^{-3}$. By (3.5) we have

$$
\left\|\mathscr{S}_{\Omega, \Psi} f\right\|_{p} \leq \sum_{\mu \in \mathbf{J} \cup\{0\}}\left|\lambda_{\mu}\right|\left\|\mathscr{S}_{\tilde{b}_{\mu}, \Psi} f\right\|_{p} .
$$

Thus, by (3.6), Theorem 1.1 (a) is proved if we can show that

$$
\left\|\mathscr{S}_{\tilde{b}_{\mu}, \Psi} f\right\|_{p} \leq C_{p}\left(\log \left|I_{\mu}\right|^{-1}\right)^{1 / 2}\|f\|_{p}
$$

for each $\mu \in \mathbf{J} \cup\{0\}, 2 \leq p<\infty$ and for some positive constant $C_{p}$ independent of $\mu$. To this end, we let $\left\{\varphi_{k, \mu}\right\}_{-\infty}^{\infty}$ be a smooth partition of unity in $(0, \infty)$ adapted to the intervals $\left[\omega_{\mu}^{-k d-|d|}, \omega_{\mu}^{-k d+|d|}\right]$. To be precise, we require the following:

$$
\begin{array}{r}
\varphi_{k, \mu} \in C^{\infty}, \quad 0 \leq \varphi_{k, \mu} \leq 1, \quad \sum_{k} \varphi_{k, \mu}(t)=1, \\
\operatorname{supp} \varphi_{k, \mu} \subseteq\left[\omega_{\mu}^{-k d-|d|}, \omega_{\mu}^{-k d+|d|}\right], \quad\left|\frac{d^{s} \varphi_{k, \mu}(t)}{d t^{s}}\right| \leq \frac{C_{s}}{t^{s}},
\end{array}
$$

where $C_{s}$ is independent of the lacunary sequence $\left\{\omega_{\mu}^{k}: k \in \mathbf{Z}\right\}$. Define the partial sum operators $S_{k, \mu}$ on $\mathbf{R}^{n}$ by

$$
\left.\widehat{\left(\widehat{S_{k, \mu}} f\right.}\right)(\xi)=\varphi_{k, \mu}(|\xi|) \hat{f}(\xi) .
$$

Since $f(x)=\sum_{k \in \mathbf{Z}}\left(S_{k+l, \mu} f\right)(x)$ for any $f \in \mathscr{S}\left(\mathbf{R}^{n}\right)$ and $l \in \mathbf{Z}$, by duality and applying Minkowski's inequality we get

$$
\begin{aligned}
\mathscr{S}_{\tilde{b}_{\mu}, \Psi} f(x) & \leq\left(\int_{0}^{\infty}\left|\int_{\mathbf{S}^{n-1}} f(x-\Psi(t) \xi) \tilde{b}_{\mu}(\xi) d \sigma(\xi)\right|^{2} \frac{d t}{t}\right)^{1 / 2} \\
& =\left(\sum_{k \in \mathbf{Z}} \int_{\omega_{\mu}^{k}}^{\omega_{\mu}^{k+1}}\left|\int_{\mathbf{S}^{n-1}} f(x-\Psi(t) \xi) \tilde{b}_{\mu}(\xi) d \sigma(\xi)\right|^{2} \frac{d t}{t}\right)^{1 / 2} \\
& =\left(\sum_{k \in \mathbf{Z}} \int_{\omega_{\mu}^{k}}^{\omega_{\mu}^{k+1}}\left|\sum_{l \in \mathbf{Z}} E_{k+l, t, \tilde{b}_{\mu}} f(x)\right|^{2} \frac{d t}{t}\right)^{1 / 2} \\
& \leq \sum_{l \in \mathbf{Z}}\left(\sum_{k \in \mathbf{Z}} \int_{\omega_{\mu}^{k}}^{\omega_{\mu}^{k+1}}\left|E_{k+l, t, \tilde{b}_{\mu}} f(x)\right|^{2} \frac{d t}{t}\right)^{1 / 2}
\end{aligned}
$$

where

$$
E_{l, t, \Omega} f(x)=\int_{\mathbf{S}^{n-1}} \Omega(\xi)\left(S_{l, \mu} f\right)(x-\Psi(t) \xi) d \sigma(\xi) .
$$

Now if we let 


$$
T_{l, \mu, \tilde{b}_{\mu}} f(x)=\left(\sum_{k \in \mathbf{Z}} \int_{\omega_{\mu}^{k}}^{\omega_{\mu}^{k+1}}\left|E_{k+l, t, \tilde{b}_{\mu}} f(x)\right|^{2} \frac{d t}{t}\right)^{1 / 2},
$$

then we have

$$
\mathscr{S}_{\tilde{b}_{\mu}, \Psi} f(x) \leq \sum_{l \in \mathbf{Z}} T_{l, \mu, \tilde{b}_{\mu}} f(x) .
$$

Therefore, to prove (3.7), it suffices to prove

$$
\left\|T_{l, \mu, \tilde{b}_{\mu}}(f)\right\|_{p} \leq C_{p}\left(\log \left|I_{\mu}\right|^{-1}\right)^{1 / 2} 2^{-\theta_{p}|l||d|}\|f\|_{p}
$$

for some positive constants $C_{p}, \theta_{p}$ and for all $2 \leq p<\infty$.

To prove (3.9), let us first compute the $L^{2}$ norm of $T_{l, \mu, \tilde{b}_{\mu}}(f)$. By Plancherel's theorem and using Lemma 2.4 we obtain

$$
\begin{aligned}
\left\|T_{l, \mu, \tilde{b}_{\mu}}(f)\right\|_{2}^{2} & =\int_{\mathbf{R}^{n}} \sum_{k \in \mathbf{Z}} \int_{\omega_{\mu}^{k}}^{\omega_{\mu}^{k+1}}\left|E_{k+l, t, \tilde{b}_{\mu}} f(x)\right|^{2} \frac{d t}{t} d x \\
& \leq \sum_{k \in \mathbf{Z}} \int_{\Delta_{k+l}}\left|Y_{\mu, k}(\xi)\right|^{2}|\hat{f}(\xi)|^{2} d \xi \\
& \leq C\left(\log \left|I_{\mu}\right|^{-1}\right) 2^{-2 \alpha|l||d|} \sum_{k \in \mathbf{Z}} \int_{\Delta_{k+l}}|\hat{f}(\xi)|^{2} d \xi \\
& \leq C\left(\log \left|I_{\mu}\right|^{-1}\right) 2^{-2 \alpha|l||d|}\|f\|_{2}^{2},
\end{aligned}
$$

where $Y_{\mu, k}(\xi)$ is defined as in Lemma 2.4 and

$$
\Delta_{k}=\left\{\xi \in \mathbf{R}^{n}: \omega_{\mu}^{-k d-|d|} \leq|\xi| \leq \omega_{\mu}^{-k d+|d|}\right\} .
$$

Therefore, we have

$$
\left\|T_{l, \mu, \tilde{b}_{\mu}}(f)\right\|_{2} \leq C\left(\log \left|I_{\mu}\right|^{-1}\right)^{1 / 2} 2^{-\alpha|l||d|}\|f\|_{2} .
$$

Now, let us compute the $L^{p}$-norm of $T_{l, \mu, \tilde{b}_{\mu}}(f)$ for $p>2$. By duality, there is a function $g$ in $L^{(p / 2)^{\prime}}\left(\mathbf{R}^{n}\right)$ with $\|g\|_{(p / 2)^{\prime}} \leq 1$ such that

$$
\begin{aligned}
\left\|T_{l, \mu, \tilde{b}_{\mu}}(f)\right\|_{p}^{2} & =\sum_{k \in \mathbf{Z}} \int_{\mathbf{R}^{n}} \int_{\omega_{\mu}^{k}}^{\omega_{\mu}^{k+1}}\left|E_{k+l, t, \tilde{b}_{\mu}} f(x)\right|^{2} \frac{d t}{t}|g(x)| d x \\
& \leq C \sum_{k \in \mathbf{Z}} \int_{\mathbf{R}^{n}} \int_{\omega_{\mu}^{k}}^{\omega_{\mu}^{k+1}} \int_{\mathbf{S}^{n-1}}\left|\tilde{b}_{\mu}(\xi)\right|\left|S_{k+l, \mu} f(x)\right|^{2}|g(x+\Psi(t) \xi)| d \sigma(\xi) \frac{d t}{t} d x \\
& \leq C \sum_{k \in \mathbf{Z}} \int_{\mathbf{R}^{n}}\left|S_{k+l, \mu} f(x)\right|^{2} v_{\mu}^{*}(\tilde{g})(-x) d x, \quad \text { where } \tilde{g}(x)=g(-x) \\
& \leq C\left\|\sum_{k \in \mathbf{Z}}\left|S_{k+l, \mu} f\right|^{2}\right\|_{(p / 2)}\left\|v_{\mu}^{*}(\tilde{g})\right\|_{(p / 2)^{\prime}} \cdot
\end{aligned}
$$


Thus, using Lemma 2.6, the Littlewood-Paley theory and Theorem 3 along with the remark that follows its statement in [St1], p. 96, we have

$$
\left\|T_{l, \mu, \tilde{b}_{\mu}}(f)\right\|_{p} \leq C_{p}\left(\log \left|I_{\mu}\right|^{-1}\right)^{1 / 2}\|f\|_{p} \text { for } 2 \leq p<\infty .
$$

Interpolating between (3.10) and (3.11) we get (3.9) which in turn ends the proof of Theorem 1.1 (a).

Proof of Theorem 1.1 (b). Assume that $\Omega \in L^{q}\left(\mathbf{S}^{n-1}\right)$ for some $q>1$. By Theorem 1.1 (a) and since for any fixed $r>1, B_{r}^{(0,-1 / 2)}\left(\mathbf{S}^{n-1}\right) \supset L^{q}\left(\mathbf{S}^{n-1}\right)$ for all $q>1$, we need to prove Theorem 1.1 (b) only for $\Omega \in L^{q}\left(\mathbf{S}^{n-1}\right)$ for some $q>1$ and $2 n \delta /(2 n+n \delta-2)<p<2$. By the same arguments employed in the proof of Theorem 1.1 (a), it suffices to show that

$$
\left\|T_{l, 0, \Omega}(f)\right\|_{p} \leq C_{p}\|f\|_{p} \quad \text { for } 2 n \delta /(2 n+n \delta-2)<p<2 \text { and } q>1 .
$$

By definition of $T_{l, 0, \Omega}$ and by a simple change of variable we have

$$
T_{l, 0, \Omega} f(x) \leq\left(\sum_{k \in \mathbf{Z}} \int_{1}^{2}\left|F_{k, l, t} f(x)\right|^{2} \frac{d t}{t}\right)^{1 / 2},
$$

where

$$
F_{k, l, t} f(x)=\int_{\mathbf{S}^{n-1}} \Omega(\xi)\left(S_{k+l, 0} f\right)\left(x-\Psi\left(2^{k} t\right) \xi\right) d \sigma(\xi) .
$$

We notice that, to prove $T_{l, 0, \Omega}(f) \in L^{p}\left(\mathbf{R}^{n}\right)$, it suffices to show that $F_{k, l, t} f(x) \in$ $L^{p}\left(l^{2}\left[L^{2}\left([1,2], \frac{d t}{t}\right), k\right], d x\right)$. By duality, there is a function $g=g_{k}(x, t)$ satisfying $\|g\| \leq 1$ and

$$
g \in L^{p^{\prime}}\left(l^{2}\left[L^{2}\left([1,2), \frac{d t}{t}\right), k\right], d x\right)
$$

such that

$$
\begin{aligned}
& \left\|T_{l, 0, \Omega}(f)\right\|_{p} \\
& \quad=\int_{\mathbf{R}^{n}} \sum_{k \in \mathbf{Z}} \int_{1}^{2} \int_{\mathbf{S}^{n-1}} \Omega(\xi) g_{k}(x, t)\left(S_{k+l, 0} f\right)\left(x-\Psi\left(2^{k} t\right) \xi\right) d \sigma(\xi) \frac{d t}{t} d x \\
& \quad=\int_{\mathbf{R}^{n}} \sum_{k \in \mathbf{Z}} \int_{1}^{2} \int_{\mathbf{S}^{n-1}}\left(S_{k+l, 0} f\right)(x) \Omega(\xi) g_{k}\left(x+\Psi\left(2^{k} t\right) \xi, t\right) d \sigma(\xi) \frac{d t}{t} d x \\
& \quad \leq\left\|(X(g))^{1 / 2}\right\|_{p^{\prime}}\left\|\left(\sum_{k \in \mathbf{Z}}\left|S_{k+l, 0} f\right|^{2}\right)^{1 / 2}\right\|_{p},
\end{aligned}
$$


where

$$
X(g)(x)=\sum_{k \in \mathbf{Z}}\left(\int_{1}^{2} \int_{\mathbf{S}^{n-1}} \Omega(\xi) g_{k}\left(x+\Psi\left(2^{k} t\right) \xi, t\right) d \sigma(\xi) \frac{d t}{t}\right)^{2} .
$$

By the Littlewood-Paley theory we have

$$
\left\|T_{l, 0, \Omega}(f)\right\|_{p} \leq C_{p}\|f\|_{p}\left\|(X(g))^{1 / 2}\right\|_{p^{\prime}} .
$$

Since $\left\|(X(g))^{1 / 2}\right\|_{p^{\prime}}=\|X(g)\|_{p^{\prime} / 2}^{1 / 2}$ and $p^{\prime}>2$, there is a function $a \in L^{\left(p^{\prime} / 2\right)^{\prime}}\left(\mathbf{R}^{n}\right)$ such that $\|a\|_{\left(p^{\prime} / 2\right)^{\prime}} \leq 1$ and

$$
\|X(g)\|_{p^{\prime} / 2}=\int_{\mathbf{R}^{n}} \sum_{k \in \mathbf{Z}}\left(\int_{1}^{2} \int_{\mathbf{S}^{n-1}} \Omega(\xi) g_{k}\left(x+\Psi\left(2^{k} t\right) \xi, t\right) d \sigma(\xi) \frac{d t}{t}\right)^{2}|a(x)| d x .
$$

Now, we need to consider two cases:

CASE 1. $2 n \delta /(2 n+n \delta-2)<p<2$ and $q \geq 2$. In this case we have $2 n /(2 n-1)<p<2$.

By Hölder's inequality, we have

$$
\begin{aligned}
& \left(\int_{1}^{2} \int_{\mathbf{S}^{n-1}} \Omega(\xi) g_{k}\left(x+\Psi\left(2^{k} t\right) \xi, t\right) d \sigma(\xi) \frac{d t}{t}\right)^{2} \\
& \quad \leq\|\Omega\|_{q}^{2} \int_{1}^{2}\left(\int_{\mathbf{S}^{n-1}}\left|g_{k}\left(x+\Psi\left(2^{k} t\right) \xi, t\right)\right|^{q^{\prime}} d \sigma(\xi)\right)^{2 / q^{\prime}} \frac{d t}{t} \\
& \quad \leq\|\Omega\|_{q}^{2} \int_{1}^{2} \int_{\mathbf{S}^{n-1}}\left|g_{k}\left(x+\Psi\left(2^{k} t\right) \xi, t\right)\right|^{2} d \sigma(\xi) \frac{d t}{t}
\end{aligned}
$$

By (3.13) and a change of variable we get

$$
\begin{aligned}
& \|X(g)\|_{p^{\prime} / 2} \\
& \quad \leq \int_{\mathbf{R}^{n}} \sum_{k \in \mathbf{Z}} \int_{1}^{2}\left|g_{k}(x, t)\right|^{2}\left(\int_{\mathbf{S}^{n-1}}\left|a\left(x+\Psi\left(2^{k} t\right) \xi\right)\right| d \sigma(\xi)\right) \frac{d t}{t} d x \\
& \quad \leq \int_{\mathbf{R}^{n}}\left(\sum_{k \in \mathbf{Z}} \int_{1}^{2}\left|g_{k}(x, t)\right|^{2} \frac{d t}{t}\right) \mathscr{M}_{S}(|a|)(x) d x .
\end{aligned}
$$

By noticing that $\left(p^{\prime} / 2\right)^{\prime}>n /(n-1)$, applying Hölder's inequality, the choice of $a$ and Lemma 2.7 we get

$$
\|X(g)\|_{p^{\prime} / 2} \leq C
$$

which in turn gives (3.12) for $2 n \delta /(2 n+n \delta-2)<p<2$ and $q \geq 2$.

CASE 2. $2 n \delta /(2 n+n \delta-2)<p<2$ and $1<q<2$.

By Hölder's inequality, Fubini's theorem and a change of variable we have 


$$
\begin{aligned}
& \|X(g)\|_{p^{\prime} / 2} \\
& \quad \leq\|\Omega\|_{q}^{q} \int_{\mathbf{R}^{n}} \sum_{k \in \mathbf{Z}} \int_{1}^{2} \int_{\mathbf{S}^{n-1}}|\Omega(\xi)|^{2-q}\left|g_{k}\left(x+\Psi\left(2^{k} t\right) \xi, t\right)\right|^{2} d \sigma(\xi) \frac{d t}{t}|a(x)| d x \\
& \quad \leq C \int_{\mathbf{R}^{n}} \sum_{k \in \mathbf{Z}} \int_{1}^{2}\left|g_{k}(x, t)\right|^{2}\left(\int_{\mathbf{S}^{n-1}}|\Omega(\xi)|^{2-q}\left|a\left(x+\Psi\left(2^{k} t\right) \xi\right)\right| d \sigma(\xi)\right) \frac{d t}{t} d x \\
& \quad \leq C \int_{\mathbf{R}^{n}} \sum_{k \in \mathbf{Z}} \int_{1}^{2}\left|g_{k}(x, t)\right|^{2}\left(\int_{\mathbf{S}^{n-1}}\left|a\left(x+\Psi\left(2^{k} t\right) \xi\right)\right|^{q^{\prime} / 2} d \sigma(\xi)\right)^{2 / q^{\prime}} \frac{d t}{t} d x \\
& \quad \leq C \int_{\mathbf{R}^{n}}\left(\sum_{k \in \mathbf{Z}} \int_{1}^{2}\left|g_{k}(x, t)\right|^{2} \frac{d t}{t}\right)\left(\mathscr{M}_{S}\left(|a|^{q^{\prime} / 2}\right)(x)\right)^{2 / q^{\prime}} d x \\
& \quad \leq C\left\|\left(\sum_{k \in \mathbf{Z}} \int_{1}^{2}\left|g_{k}(x, t)\right|^{2} \frac{d t}{t}\right)^{1 / 2}\right\|\left\|_{p^{\prime} / 2}\right\|\left(\mathscr{M}_{S}\left(|a|^{q^{\prime} / 2}\right)\right)^{2 / q^{\prime}} \|_{\left(p^{\prime} / 2\right)^{\prime}} .
\end{aligned}
$$

Since $\left(2 / q^{\prime}\right)\left(p^{\prime} / 2\right)^{\prime}>n /(n-1)$, by Lemma 2.7 we get

$$
\|X(h)\|_{p^{\prime} / 2} \leq C
$$

which implies (3.12) for $2 n \delta /(2 n+n \delta-2)<p<2$ and $1<q<2$. This completes the proof of Theorem 1.2 (b).

Proof of Theorem 1.1 (c). By duality, the operator $\mathscr{S}_{\Omega}$ is simply

$$
\mathscr{S}_{\Omega} f(x)=\left(\int_{0}^{\infty}\left|\int_{\mathbf{S}^{n-1}} f(x-t y) \Omega(y) d \sigma(y)\right|^{2} \frac{d t}{t}\right)^{1 / 2} .
$$

It is obvious that $\mathscr{S}_{\Omega}$ is bounded on $L^{2}\left(\mathbf{R}^{n}\right)$ if and only if the multiplier

$$
m(\xi)=\left(\int_{0}^{\infty}\left|\int_{\mathbf{S}^{n-1}} e^{-2 \pi i t \xi \cdot x} \Omega(x) d \sigma(x)\right|^{2} \frac{d t}{t}\right)^{1 / 2}
$$

is an $L^{\infty}$ function. It is easy to see that

$$
m(\xi)=\lim _{N \rightarrow \infty, \varepsilon \rightarrow 0} \int_{\mathbf{S}^{n-1} \times \mathbf{S}^{n-1}} \Omega(x) \overline{\Omega(y)} \int_{\varepsilon|\xi|}^{N|\xi|}\left(e^{-2 \pi i t \xi^{\prime} \cdot(x-y)} \frac{d t}{t}\right) d \sigma(x) d \sigma(y) .
$$

Notice that

$$
\int_{\varepsilon|\xi|}^{N|\xi|}\left(e^{-2 \pi i t \xi^{\prime} \cdot(x-y)}-\cos (2 \pi t)\right) \frac{d t}{t} \rightarrow \log \left|\xi^{\prime} \cdot(x-y)\right|^{-1}-i \frac{\pi}{2} \operatorname{sgn}\left(\xi^{\prime} \cdot(x-y)\right)
$$

as $N \rightarrow \infty$ and $\varepsilon \rightarrow 0$, and the integral is bounded, uniformly in $\varepsilon$ and $N$, $C\left(1+\log \left|\xi^{\prime} \cdot(x-y)\right|\right)$. Therefore, using (1.1) and the Lebesgue dominated convergence theorem we obtain 


$$
m(\xi)=\int_{\mathbf{S}^{n-1} \times \mathbf{S}^{n-1}} \Omega(x) \overline{\Omega(y)}\left(\log \left|\xi^{\prime} \cdot(y-x)\right|^{-1}-i \frac{\pi}{2} \operatorname{sgn}\left(\xi^{\prime} \cdot(y-x)\right)\right) d \sigma(x) d \sigma(y) .
$$

Now, if $\Omega$ is a real-valued function, we have

$$
m(\xi)=\int_{\mathbf{S}^{n-1} \times \mathbf{S}^{n-1}} \Omega(x) \Omega(y)\left(\log \left|\xi^{\prime} \cdot(x-y)\right|^{-1}\right) d \sigma(x) d \sigma(y) .
$$

Now, we are ready to prove part (c) of Theorem 1.1. For the sake of simplicity we shall present the construction of $\Omega$ only in the case $n=2$ and $q=\infty$. Other cases can be obtained by minor modifications. Also, we shall work on $[-1,1]$ instead of $\mathbf{S}^{1}$. We follow a similar argument as in [AA2]. For $x \in[-1,1]$, let

$$
\Omega(x)=\sum_{k=1}^{\infty} \lambda_{k} b_{k}(x)
$$

where $I_{k}=\left[\frac{1}{k+1}, \frac{1}{k}\right)$ for $k \geq 2$,

$$
\begin{aligned}
& \lambda_{1}=\sum_{k=2}^{\infty} \frac{1}{(k+1)(\log k)^{3 / 2}}, \quad b_{1}(x)=-\chi_{[-1,0]}(x), \\
& \lambda_{k}=\frac{1}{(k+1)(\log k)^{3 / 2}}, \quad b_{k}(x)=\left|I_{k}\right|^{-1} \chi_{I_{k}}(x) .
\end{aligned}
$$

Then $\Omega$ has the desired properties. More precisely, $\Omega$ satisfies the following:

$$
\begin{gathered}
\int_{-1}^{1} \Omega(x) d x=0 ; \\
\Omega \in B_{\infty}^{(0, v)}([-1,1]) \text { for each } v,-1<v<-\frac{1}{2} ; \\
\Omega \notin B_{\infty}^{(0,-1 / 2)}([-1,1]) ; \\
S_{1}=\int_{[0,1]^{2}}\left(\Omega(x) \Omega(y) \log |x-y|^{-1}\right) d x d y=\infty ; \\
S_{2}=\int_{[-1,1]^{2} \backslash[0,1]^{2}}|\Omega(x) \Omega(y) \log | x-\left.y\right|^{-1} \mid d x d y<\infty .
\end{gathered}
$$

The proof of (3.16)-(3.17) is straightforward. Now we turn to the proof of (3.18). We first notice that each $b_{k}$ is an $\infty$-block supported on the interval $I_{k}$. So to prove (3.18), we only need to show that $N_{\infty}^{(0,-1 / 2)}(\Omega)=\infty$. To this end, by Lemma 2.3 we have for each $l$, 


$$
\begin{aligned}
N_{\infty}^{(0,-1 / 2)}\left(\Omega+\lambda_{1} \chi_{[-1,0]}\right) & \geq \sum_{k=2}^{m}\left|\lambda_{k}\right|\left|I_{k}\right|^{-1} N_{\infty}^{(0,-1 / 2)}\left(\chi_{I_{k}}\right) \\
& \geq \sum_{k=2}^{m}\left|\lambda_{k}\right|\left(1+\log ^{1 / 2}\left(\left|I_{k}\right|^{-1}\right)\right) .
\end{aligned}
$$

Letting $m \rightarrow \infty$, we get $N_{\infty}^{(0,-1 / 2)}\left(\Omega+\lambda_{1} \chi_{[-1,0]}\right)=\infty$. Since, $N_{\infty}^{(0,-1 / 2)}\left(\lambda_{1} \chi_{[-1,0]}\right)$ $<\infty$ we get $N_{\infty}^{(0,-1 / 2)}(\Omega)=\infty$.

Now, we verify (3.19). Notice that for $(x, y) \in I_{k} \times I_{j}$, we have $\log |x-y|^{-1}$ $\geq 0$. Therefore, we have

$$
S_{1} \geq \sum_{j=2}^{\infty} \sum_{k=2(j+1)}^{\infty} \frac{k j}{(\log k)^{3 / 2}(\log j)^{3 / 2}} \int_{I_{k} \times I_{j}} \log |x-y|^{-1} d x d y .
$$

We notice that, for each $(x, y) \in I_{k} \times I_{j}$ with $k \geq 2(j+1)$, we have $y \geq 2 x$. Thus $|x-y|=y-x \leq \frac{1}{j}$ and so $\log |x-y|^{-1} \geq \log j$ which in turn leads to

$$
\begin{aligned}
S_{1} & \geq C \sum_{j=2}^{\infty} \frac{1}{j(\log j)^{1 / 2}}\left(\sum_{k=2(j+1)}^{\infty} \frac{1}{k(\log k)^{3 / 2}}\right) \\
& \geq C \sum_{j=2}^{\infty} \frac{1}{j(\log j)}=\infty .
\end{aligned}
$$

Finally, we verify (3.20). To this end, we divide the integral domain $[-1,1]^{2} \backslash[0,1]^{2}$ into three parts: $[-1,0] \times[0,1],[0,1] \times[-1,0]$, and $[-1,0] \times$ $[-1,0]$. First, the integral over $[-1,0] \times[0,1]$ is dominated from above by

$$
C \sum_{k=2}^{\infty} \frac{k}{(\log k)^{3 / 2}}\left|\int_{I_{k}} \int_{-1}^{0} \log \right| x-\left.y\right|^{-1} d x d y \mid .
$$

By straightforward calculations, we have

$$
\left|\int_{I_{k}} \int_{-1}^{0}\left(\log |x-y|^{-1}\right) d x d y\right| \leq C \frac{1}{k^{2}}
$$

for some positive constant independent of $k$. Thus, we have

$$
C \sum_{k=2}^{\infty} \frac{k}{(\log k)^{3 / 2}}\left|\int_{I_{k}} \int_{-1}^{0} \log \right| x-\left.y\right|^{-1} d x d y \mid \leq C \sum_{k=2}^{\infty} \frac{1}{k(\log k)^{3 / 2}}<\infty .
$$

Similarly, the integral over $[0,1] \times[-1,0]$ is finite. Finally, the integral over $[-1,0] \times[-1,0]$ is finite because $\left(\sum_{k=2}^{\infty} \frac{1}{(k+1)(\log k)^{3 / 2}}\right)^{2} \chi_{[-1,0] \times[-1,0]} \in L^{\infty}$. This finishes the proof of Theorem 1.1 (c). 


\section{A further result}

In this section, we are concerned with a maximal operator related to the Marcinkiewicz integral operator $\mu_{\Omega, h}$ which is defined by

$$
\mu_{\Omega, h} f(x)=\left(\int_{0}^{\infty}\left|\int_{|y| \leq t} f(x-y) \frac{\Omega(y /|y|)}{|y|^{n-1}} h(|y|) d y\right|^{2} \frac{d t}{t^{3}}\right)^{1 / 2},
$$

where $h$ is a measurable function on $\mathbf{R}^{+}$and $\Omega \in L^{1}\left(\mathbf{S}^{n-1}\right)$ is a function satisfying (1.1).

Marcinkiewicz integral operators have been investigated by many authors, dating back to the investigations of such operators by A. Zygmund on the circle and E. Stein on $\mathbf{R}^{n}$. For a sampling of past studies, see [St2], [BCP], [Wa], [AACP] and [AA2]. In particular, Al-Qassem and Al-Salman in [AA2] showed that $\mu_{\Omega, 1}$ is bounded on $L^{p}\left(\mathbf{R}^{n}\right)(1<p<\infty)$ if $\Omega \in B_{q}^{(0,-1 / 2)}\left(\mathbf{S}^{n-1}\right)$ and there exists an $\Omega$ which lies in $B_{q}^{(0, v)}\left(\mathbf{S}^{n-1}\right)$ for all $-1<v<-\frac{1}{2}$ such that $\mu_{\Omega, 1}$ is not bounded on $L^{2}\left(\mathbf{R}^{n}\right)$.

Motivated by the definition of $\mathscr{S}_{\Omega}$, a maximal operator $S_{\Omega}$ corresponding to $\mu_{\Omega, h}$ can be defined by

$$
\mathscr{M}_{\Omega} f(x)=\sup _{h \in \mathscr{H}}\left(\int_{0}^{\infty}\left|\int_{|y| \leq t} f(x-y) \frac{\Omega(y /|y|)}{|y|^{n-1}} h(|y|) d y\right|^{2} \frac{d t}{t^{3}}\right)^{1 / 2},
$$

where $\Omega \in L^{1}\left(\mathbf{S}^{n-1}\right)$ is a function satisfies the cancellation condition (1.1).

We have the following result concerning this maximal operator $\mathscr{M}_{\Omega}$ :

THEOREM 4.1. Let $n \geq 2$ and $\mathscr{M}_{\Omega}$ be given as in (4.1). Then

(a) If $\Omega \in B_{q}^{(0,-1 / 2)}\left(\mathbf{S}^{n-1}\right)$ (for some $q>1$ ) and satisfies (1.1), then $\mathscr{M}_{\Omega}$ is bounded on $L^{p}\left(\mathbf{R}^{n}\right)$ for $2 \leq p<\infty$;

(b) If $\Omega \in L^{q}\left(\mathbf{S}^{n-1}\right)$ (for some $q>1$ ) and satisfies (1.1), then $\mathscr{M}_{\Omega}$ is bounded on $L^{p}\left(\mathbf{R}^{n}\right)$ for $2 n \delta /(2 n+n \delta-2)<p<\infty$, where $\delta=\max \left\{2, q^{\prime}\right\}$.

Proof. By Minkowski's inequality we obtain

$$
\begin{aligned}
& \left(\int_{0}^{\infty}\left|\int_{|y| \leq t} f(x-y) \frac{\Omega\left(y^{\prime}\right)}{|y|^{n-1}} h(|y|) d y\right|^{2} \frac{d t}{t^{3}}\right)^{1 / 2} \\
& \quad \leq\left(\int_{0}^{\infty}\left(\int_{0}^{\infty}\left|\int_{\mathbf{S}^{n-1}} f(x-s y) \Omega(y) d \sigma(y)\right||h(s)| \chi_{[0, t]}(s) d s\right)^{2} \frac{d t}{t^{3}}\right)^{1 / 2} \\
& \quad \leq \int_{0}^{\infty}\left(\int_{0}^{\infty}\left|\int_{\mathbf{S}^{n-1}} f(x-s y) \Omega(y) d \sigma(y)\right|^{2}|h(s)|^{2} \chi_{[0, t]}(s) \frac{d t}{t^{3}}\right)^{1 / 2} d s
\end{aligned}
$$




$$
\begin{aligned}
& =\int_{0}^{\infty}\left|\int_{\mathbf{S}^{n-1}} f(x-s y) \Omega(y) d \sigma(y)\right||h(s)|\left(\int_{s}^{\infty} \frac{d t}{t^{3}}\right)^{1 / 2} d s \\
& =\frac{1}{\sqrt{2}} \int_{0}^{\infty}\left|\int_{\mathbf{S}^{n-1}} f(x-s y) \Omega(y) d \sigma(y)\right||h(s)| \frac{d s}{s} .
\end{aligned}
$$

Thus, by Hölder's inequality we have

$$
\mathscr{M}_{\Omega} f(x) \leq \frac{1}{\sqrt{2}}\left(\int_{0}^{\infty}\left|\int_{\mathbf{S}^{n-1}} f(x-s y) \Omega(y) d \sigma(y)\right|^{2} \frac{d s}{s}\right)^{1 / 2}=\frac{1}{\sqrt{2}} \mathscr{S}_{\Omega} f(x) .
$$

Therefore, Theorem 4.1 follows immediately from by Theorem 1.1.

\section{REFERENCES}

[AA1] H. Al-QASSEm AND A. Al-SAlman, $L^{p}$ boundedness of a class of singular integral operators with rough kernels, Turkish J. Math. 25 (2001), 519-533.

[AA2] H. Al-Qhssem and A. Al-Salman, A note on Marcinkiewicz integral operators, J. Math. Anal. Appl. 282 (2003), 698-710.

[AacP] A. Al-Salman, H. Al-Qassem, L. Cheng and Y. Pan, $L^{p}$ bounds for the function of Marcinkiewicz, Math. Res. Lett. 9 (2002), 697-700.

[AAP1] H. Al-QAssem, A. Al-Salman AND Y. PAN, Singular integrals associated to homogeneous mappings with rough kernels, Hokkaido Math. J. 33 (2004), 551-569.

[AAP2] H. Al-QASsem, A. Al-Salman and Y. Pan, Rough singular integrals with kernels supported by submanifolds of finite type, Asian J. Math. 9 (2005), 19-30.

[AP] H. AL-QASSEm AND Y. PAN, $L^{p}$ estimates for singular integrals with kernels belonging to certain block spaces, Revista Matemática Iberoamericana 18 (2002), 701-730.

[BCP $]$ A. Benedek, A. Calderón and R. Panzone, Convolution operators on Banach space valued functions, Proc. Nat. Acad. Sci. U.S.A. 48 (1962), 356-365.

[Bo] J. Bourgain, Average in the plane over convex curves and maximal operators, J. Analyse Math. 47 (1986), 69-85.

[CZ] A. P. Calderón And A. Zygmund, On singular integrals, Amer. J. Math. 78 (1956), 289309.

[CL] L. K. Chen And H. Lin, A maximal operator related to a class of singular integrals, Illi. Jour. Math. 34 (1990), 120-126.

[CW] R. Coifman and G. Weiss, Extension of Hardy spaces and their use in analysis, Bull. Amer. Math. Soc. 83 (1977), 569-645.

[Co] W. C. Connetr, Singular integrals near $L^{1}$, Proc. Sympos. Pure. Math. (S. Wainger and G. Weiss, eds.) 35, Amer. Math. Soc., Providence, RI, 1979, 163-165.

[DR] J. Duonndikoetxea and J. L. Rubio de Francia, Maximal functions and singular integral operators via Fourier transform estimates, Invent. Math. 84 (1986), 541-561.

[Fe] R. Fefferman, A note on singular integrals, Proc. Amer. Math. Soc. 74 (1979), 266-270.

[KS] M. Keitoku and E. Sato, Block spaces on the unit sphere in $\mathbf{R}^{n}$, Proc. Amer. Math. Soc. 119 (1993), 453-455.

[LTW] S. Lu, M. Taibleson and G. Weiss, Spaces generated by blocks, Beijing Normal University Press, Beijing, 1989.

[St1] E. M. SteIn, On the functions of Littlewood-Paley, Lusin and Marcinkiewicz, Trans. Amer. Math. Soc. 88 (1958), 430-466. 
[St2] E. M. Stein, Singular integrals and differentiability properties of functions, Princeton University Press, Princeton, NJ, 1970.

[St3] E. M. SteIN, Maximal functions: spherical means, Proc. Nat. Acad. Sci. USA 73 (1976), 2174-2175.

[St4] E. M. SteIn, Harmonic analysis real-variable methods, orthogonality and oscillatory integrals, Princeton University Press, Princeton, NJ, 1993

[TW] M. H. Taibleson AND G. Weiss, Certain function spaces associated with a.e. convergence of Fourier series, Univ. of Chicago Conf. in honor of Zygmund, Woodsworth, 1983.

[Wa] T. WALsh, On the function of Marcinkiewicz, Studia Math. 44 (1972), 203-217.

Department of Mathematics

YARMOUK UNIVERSITY

IRBID

JORDAN

E-mail: husseink@yu.edu.jo 